\title{
Description of Tramadol Mortality in Osteoarthritis May Not Be Accurate
}

\section{To THE EDITOR:}

In a JAMA manuscript, Zeng et al (1), utilizing statistically complex modeling and assessment tools, concluded tramadol was associated with a significantly higher mortality compared to nonsteroidal anti-inflammatory drugs (NSAIDs). This type of assessment can only show association, rather than cause and effect relationship. While the authors acknowledge the limitations of their study, this conclusion may cause inappropriate fear and curtailing of tramadol prescriptions. This in turn may lead to increased prescriptions of NSAIDs or other opioids, potentially having the unintended consequence of increased mortality. To add to the complexity, the SPACE randomized trial (2), tramadol was considered equivalent to NSAIDs and other nonopioid drugs.

Participants in the tramadol cohort in this study had a higher prevalence of comorbidities, including diabetes, hypertension, peptic ulcer disease, chronic kidney, cardiovascular diseases and also have not considered the severity of osteoarthritis. Further, they were on a multitude of prescriptions involving numerous drugs, which may be responsible for the morbidity and mortality, including other opioids, which, curiously, were not described in this manuscript other than codeine. However, there was no significant difference in mortality compared to codeine as patients receiving codeine also had high comorbidities. The comorbidity burden with their treatments, which leads to increased levels of mortality, may not be appropriately adjusted even with such a complex statistical model. It is wellknown that oral NSAIDs, the predominant analgesic medications, and opioids commonly prescribed for managing osteoarthritis have multiple safety concerns with potential increase in mortality. Tramadol is a weak opioid agonist and is largely devoid of complications associated with NSAIDs such as serious cardiovascular and gastrointestinal adverse effects (3).

Further, the safety profile of tramadol has been shown to be similar to other opioids (4). The increase in overall mortality is clearly multifactorial and it is thus difficult to understand the role of tramadol in increasing infection, cancer, and musculoskeletal disorders related mortality. In addition, the study failed to delve into potential critical drug-drug interactions with tramadol, even though Table 2 clearly describes a significant number of patients taking serotonin modulators, which could result in adverse outcomes (5). The authors also described that $30 \%$ of patients, causes of death could not be ascertained and the study lacked adequate statistical power.

In this era of heightened opioid awareness, it is essential to precisely correlate causes of mortality. Unfortunately, this study was based on a complex statistical model, had many inconclusive findings and was statistically underpowered making it far from precise.

Laxmaiah Manchikanti, MD

Clinical Professor

Anesthesiology and Perioperative Medicine

University of Louisville, KY

Professor of Anesthesiology-Research

Department of Anesthesiology, School of Medicine LSU Health Sciences Center

Co-Director, Pain Management Centers of America

67 Lakeview Drive

Paducah, KY 42001

E-mail: drlm@thepainmd.com

Alan D. Kaye, MD, PhD

Professor, Program Director, and Chairman

Department of Anesthesiology

Louisiana State University Health Science Center

1542 Tulane Ave, Room 659

New Orleans, LA 70112

E-mail: akaye@lsuhsc.edu

Joshua A. Hirsch, MD

Vice Chair and

Service Line Chief, Interventional Radiology

Chief of Neurolnterventional Spine

Director Interventional Neuroradiology

Massachusetts General Hospital

Associate Professor of Radiology

Harvard Medical School

55 Blossom St., Gray 241B

Boston, MA 02114

E-mail: jahirsch@mgh.harvard.edu 


\section{References}

1. Zeng C, Dubreuil M, LaRochelle MR, Lu N, Wei J, Choi HK, Lei G, Zhang Y. Association of tramadol with all-cause mortality among patients with osteoarthritis. JAMA 2019; 321:969-982.

2. Krebs $E E$, Gravely $A$, Nugent $S$, Jensen AC, DeRonne B, Goldsmith ES, Kroenke K, Bair MJ, Noorbaloochi S. Effect of opioid vs nonopioid medications on pain- related function in patients with chronic back pain or hip or knee osteoarthritis pain: The SPACE randomized clinical trial. JAMA 2018; 319:872-882.

3. Katz WA. Pharmacology and clinical experience with tramadol in osteoarthritis. Drugs 1996; 52(suppl 3):39-47.

4. Solomon DH, Rassen JA, Glynn RJ, Gar- neau K, Levin R, Lee J, Schneeweiss S. The comparative safety of opioids for nonmalignant pain in older adults. Arch Intern Med 2010; 170:1979-1986.

5. Beakley BD, Kaye AM, Kaye AD. Tramadol, pharmacology, side effects and serotonin syndrome: A review. Pain Physician 2015; 18:395-400. 